\title{
End non-essential use of antimicrobials in livestock
}

\section{A rational response by $\mathrm{WHO}$ to the crisis in antimicrobial resistance}

\author{
Alison Holmes professor ${ }^{1}$, Mark Holmes reader ${ }^{2}$, Thomas Gottlieb senior specialist ${ }^{3}$, Lance B Price \\ professor $^{4}$, Arnfinn Sundsfjord professor ${ }^{5}$ \\ ${ }^{1}$ Department of Infectious Diseases and the NIHR Health Protection Research Unit in Healthcare Associated Infections and Antimicrobial Resistance, \\ Imperial College London, UK; ${ }^{2}$ Department of Veterinary Medicine, University of Cambridge, UK; ${ }^{3}$ Department of Microbiology and Infectious \\ Diseases, Concord Hospital, Sydney, Australia; ${ }^{4}$ Milken Institute School of Public Health, George Washington University, Washington DC, US; \\ ${ }^{5}$ Department of Medical Biology, Faculty of Health Sciences, University of Troms $\varnothing$-The Arctic University of Norway
}

Tackling antimicrobial resistance (AMR) is a priority of the World Health Organization's public health agenda, and in November 2017 the organisation launched guidelines on the use of medically important antimicrobials in the food production industry. Their unambiguous recommendations were that their routine use for animal growth promotion and disease prevention in healthy animals should be discontinued (Boxed Text on page 2box 1). ${ }^{1}$ In line with the global action plan on AMR, ${ }^{2}$ the aim is to help preserve the effectiveness of antimicrobials critical for human medicine. As a supranational body, WHO has taken the opportunity to put independent pressure on healthcare policy makers to now respond to its challenge. 


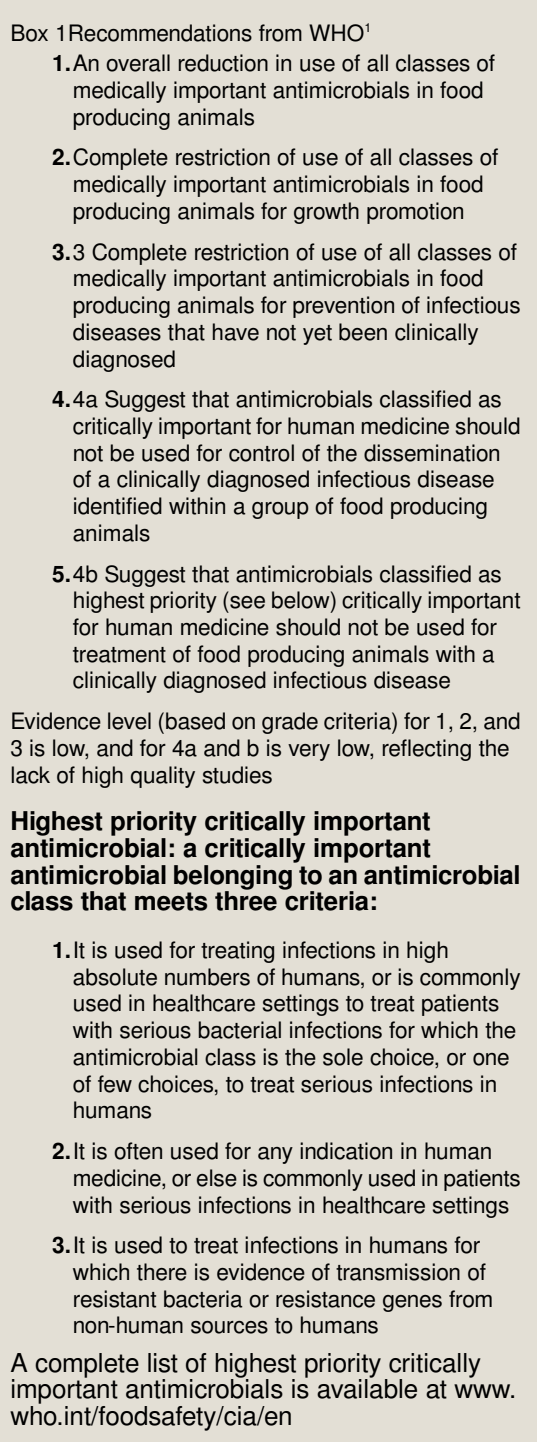

Although the guidelines were welcomed by world leaders, ${ }^{3}$ some organisations, particularly in the food producing industry, including the US Department of Agriculture, considered it to be too uncompromising and questioned the underpinning evidence. Others said the recommendations were insufficiently far reaching. A visibly more united interagency approach with the UN's Food and Agriculture Organisation and the World Organisation for Animal Health could have strengthened the message. Nonetheless, the recommendations are an important step forward.

It is widely accepted that antimicrobial exposure is the single most important driver for the emergence of, and increase in, AMR. ${ }^{4}$ Moreover, AMR bacteria or AMR-encoding genes may transfer from animals to humans through the environment, food chain, or by direct contact. Despite this, in some countries as much as $80 \%$ of the total consumption of antimicrobials is in the animal sector, with marked variation across nations. ${ }^{56}$ Within Europe, for example, use in Spain is over a 100-fold more per production unit than in Norway. ${ }^{5}$ Emerging economies are projected to increase their use of antimicrobials in livestock, contributing to a projected global increase of $67 \%$ from 2010 to $2030 .^{6}$

Some countries have already successfully reduced antimicrobial use in livestock without compromising animal health or productivity. ${ }^{17}$ The WHO guidelines do not restrict any required treatment or the targeted prevention of specific diseases. An accompanying policy brief gives guidance on implementation and actions to preserve the effectiveness of antimicrobials in veterinary medicine, including reduction targets, surveillance measures, and improving nutrition, hygiene, infection prevention, and the use of vaccines. ${ }^{1}$ Healthy animals need fewer antimicrobials but many agricultural stakeholders see the push to reduce antimicrobial consumption as unwarranted interference, rather than a drive to improve animal health. The new guidelines were shaped by published reviews commissioned by the WHO Advisory Group on Integrated Surveillance of AMR. ${ }^{89}$ This work reinforced the established understanding that reducing antimicrobial use in agriculture is associated with reductions in antimicrobial resistance in food producing animals, as well as the idea that, at minimum, the benefit appears to extend to farmers and those in direct contact with food producing animals. ${ }^{9}$ The authors clearly acknowledge the limitations in these reviews, and although there are few studies showing a direct effect on human health of antimicrobial use in animals, the overall body of evidence provided enough evidence to support WHO's recommendations.

The recommendations could have been more critical of the evidence supporting use of antimicrobials as growth promoters. ${ }^{10}$ Moreover, the focus on specific antimicrobial classes rather than a broader consideration of all antimicrobials could be questioned, as the use of one class may select for resistance to other classes, because of shared mechanisms of resistance and genetically linked resistance determinants.

AMR is complex; different bacterial species, resistance mechanisms, transferable plasmids, host populations, and environmental reservoirs contribute to direct and indirect transmission. Further studies to to characterise the spread of AMR in both animals and humans are needed, supported by more sophisticated methodology and technology.

Bacterial genome sequencing shows a complex emerging picture, with evidence of an association between human infections and reservoirs of antimicrobial resistance in animals. Heavy use of colistin in Chinese livestock and the emergence and spread of colistin resistance led to the banning of colistin for growth promotion in China in November 2016. ${ }^{811}$

But we also need to maintain perspective. Successful interventions to reduce antimicrobial use in livestock have not always resulted in AMR reduction in humans, and molecular studies of some bacterial species show that direct transmission of the organism or the resistance genes from livestock to humans may be less important than other sources, including human-to-human transmission. ${ }^{12}$ WHO guidelines highlight the need for further research to tackle knowledge gaps and improve the quality of evidence, including more standardised methodology, better surveillance, and higher quality data.

Eliminating the non-essential use of antimicrobials in livestock is a rational and welcome response to the growing crisis in AMR. The overarching aims fall within the generally agreed principles of antimicrobial stewardship by veterinarians, industry, and government. As countries are often reluctant to phase out non-essential use, whether through uncertainty or because of commercial pressures, the guidelines provide a necessary external impetus. They may also encourage societal response, consumer expectation, and civil action to drive and sustain this agenda. Of course, action in animals must not distract us from action within human healthcare-antimicrobial stewardship, effective prevention of infections, and the need for clean water and sanitation-but must been seen as an essential additional requirement in our global response to AMR. 
Competing interests: We have read and understood the BMJ policy on declaration of interests and declare that the following authors have received payments for research, expenses, or speaking engagements from drug manufacturers with an interest in antibiotics. The paid work was unrelated to the topic of this editorial MH, TG, LBP. LBP has received research support from Merck and general operating funds from Applegate Farms. LBP has received expenses for lectures from FAIRR and BlackRock LLC.

1 World Health Organization. WHO guidelines on the use of medically important antimicrobials in food producing animals. 2017. http://apps.who.int/iris/bitstream/10665/ antimicrobials in food producing anim

2 World Health Organization. Global action plan on antimicrobial resistance. 2015. www. who.int/antimicrobial-resistance/global-action-plan/en.

3 Brundtland GH. New WHO guidelines are crucial step to fighting antimicrobial resistance. BMJ Opinion. 2017. http://blogs.bmj.com/bmj/2017/12/06/gro-harlem-brundtland-newwho-guidelines-are-crucial-step-to-fighting-antimicrobial-resistance.

4 Holmes AH, Moore LSP, Sundsfjord A, et al. Understanding the mechanisms and drivers of antimicrobial resistance. Lancet 2015;6736.176-87.

5 European Medicines Agency. Sales of veterinary antimicrobial agents in 29 European countries in 2014. 2016. www.ema.europa.eu/docs/en GB/document library/Report/2016 10/WC500214217.pdf.
6 Van BoeckelTPBrowerCGilbertM, et al. Global trends in antimicrobial use in food animals. Proc Natl Acad Sci U S A2015;112:5649-54. doi:10.1073/pnas.150314111225792457

7 AarestrupFMJensenVFEmborgHDJacobsenEWegenerHC. Changes in the use of antimicrobials and the effects on productivity of swine farms in Denmark. Am J Vet Res2010;71:726-33. doi:10.2460/ajvr.71.7.72620594073

8 WebbHEAnguloFJGranierSAScottHMLoneraganGH. Illustrative examples of probable transfer of resistance determinants from food animals to humans: Streptothricins, glycopeptides, and colistin. F1000Res2017;6:1805 glycopeptides, and colistin. F1000Res2017;6:1805.

9 TangKLCaffreyNPNóbregaDB, et al. Restricting the use of antibiotics in food producing animals and its associations with antibiotic resistance in food producing animals and human beings: a systematic review and meta-analysis. Lancet Planetary Health 2017;1:e316-27.

10 CollignonPWegenerHCBraamPButlerCD. The routine use of antibiotics to promote animal growth does little to benefit protein undernutrition in the developing world. Clin Infect Dis2005;41:1007-13. doi:10.1086/43319116142667

11 LarsenJPetersenALarsenAR, et alDanish MRSA Study Group. Emergence of livestock-associated methicillin-resistant Staphylococcus aureus bloodstream infections in Denmark. Clin Infect Dis2017. doi:10.1093/cid/cix504.28575216

12 MatherAEReidSWMaskellDJ, et al. Distinguishable epidemics of multidrug-resistant Salmonella Typhimurium DT104 in different hosts. Science2013;341:1514-7. doi:10.1126/science.124057824030491

Published by the BMJ Publishing Group Limited. For permission to use (where not already granted under a licence) please go to http://group.bmj.com/group/rights-licensing/ permissions 